CLNS-99/1602, KIAS-P99006

hep-th/9901159

\title{
Membranes from Five-Branes and Fundamental Strings from $\mathbf{D} p$ Branes
}

\author{
Piljin Yi円 \\ Newman Laboratory of Nuclear Studies Cornell University \\ Ithaca, New York 14853-5001, USA \\ and \\ School of Physics, Korea Institute for Advanced Study \\ 207-43 Cheongryangri-Dong, Dongdaemun-Gu, Seoul 130-012, Korea
}

\begin{abstract}
We argue that M2 brane is realized as a topological soliton on a coincident pair of M5 and anti-M5 branes, as the two five-branes annihilate each other. Topology and quantum numbers of this world-volume soliton are discussed in some detail, and its formation is explained qualitatively. It follows from a compactification that a D4-antiD4 pair annihilate and produce type II fundamental strings. The phenomenon is best described as the confinement of a world-volume $U(1)$ gauge field on D4-anti-D4, where the confined electric flux string is identified as the fundamental string. This generalizes to other $\mathrm{D} p$-anti-D $p$ systems, and solves a puzzle recently pointed out by Witten.
\end{abstract}

\footnotetext{
${ }^{1}$ electronic mail: piljin@mail.lns.cornell.edu
} 


\section{Introduction}

The basic idea behind A. Sen's realization of supersymmetric D branes from nonsupersymmetric Dbrane-anti-D-brane system [1, 2, 3], is simple. The instability of the initial configuration manifests itself in a tachyonic mode of open string, which is charged under some world-volume gauge field. The tachyon rolls down to a true vacuum, but may create a topological defect in the process. In the simplest case of a D-anti-D pair, the broken gauge group is $U(1)$, and possible topological solitons are vortices. A. Sen identified this soliton as supersymmetric D-brane of two lower dimensions. Recently, this effect was further studied in a more abstract perspective of $K$ theory [4, 6, 6, 7].

Physics here is so simple that one naturally wonders whether a similar phenomenon should exist in other branes, say, NS5 branes and M5 branes. Type IIB NS5 branes are related to D5 branes by a U-duality, so whatever happens with D5 should happen with IIB NS5 as well. Indeed, worldvolume theory of type IIB NS5 branes is an ordinary gauge theory, and D3 branes again appear as vortex solitons. On the other hand, Type IIA NS5 branes and M5 branes share the same low energy dynamics, modulo the matter of periodicity of one of the scalar fields. We start this paper by trying to understand what happens when a M5 brane coincides with an anti-M5 brane.

M5 branes or type IIA NS5 branes admit $(2,0)$ supersymmetry, so their world-volume degrees of freedom must be in a tensor multiplet, whose bosonic field content include a chiral 2-form $B_{M N}^{+}$. Anti-M5 admits anti-chiral 2-form field $B^{-}$. On the M5-anti-M5 world-volume, the combined and unrestricted 2-form field $B$ allows a soliton of co-dimension three that carries a quantized magnetic flux $H=d B$. This object will be shown to carry membrane charge, and, in the spirit of Sen's construction, identified as M2.

This construction has an interesting corollary in the context of D branes. Note that, compactified on a circle, it reduces to a system of a D4-anti-D4 pair that annihilates and produces wrapped membranes, or equivalently fundamental strings of type IIA theory. Via T-duality transformations, it also tells us that $\mathrm{D} p$-anti- $\mathrm{D} p$ pairs annihilate and produce fundamental strings. The physics of the condensation is, it turns out, best described as the confinement of the unbroken $U(1)$ gauge 
field on $\mathrm{D} p$-anti-D $p$. 2] The fundamental string is realized as the confined electric flux string of this $U(1)$ that remains unbroken after the open string tachyon condenses.

This observation solves a puzzle which was recently posed by Witten 画, who observed that the tachyon condensation of Sen breaks only one of two $U(1)$ gauge fields on $\mathrm{D} p$-anti- $\mathrm{D} p$ world-volume, whereas both must disappear once the pair annihilate each other. We have just learned that, while the second $U(1)$ indeed remains unbroken, it is actually confined and its only remnant is the fundamental string itself.

The paper is organized as follows. In section 2, we start with a D4-anti-D4 system and explain how it naturally predicts a co-dimension-three soliton on M5-anti-M5. The topological coupling that induces the appropriate M2 charge on such solitons is identified and explained. We also discuss topology of the world-volume fields. Section 3 discusses some features of low energy dynamics that are independent of details of the condensation mechanism, and explains how the topology of the soliton is supported by the topology of the would-be Goldstone vector field. Section 4 starts by compactifying M5-anti-M5 on a circle to deduce that a fundamental string is also realized on D4-anti-D4, where the condensing tachyonic objects originate from open D2 branes. These objects are magnetically charged with respect to the overall $U(1)$ gauge field, as we will explain in detail. The resulting dual Higgs machanism generalizes to other $\mathrm{D} p$-anti-D $p$ systems. We conclude with a summary.

\section{Membranes from M5-Anti-M5}

Let us start with a system of coincident D4-anti-D4 brane pair, later to be considered as M5-antiM5 that are wrapped along the compact circle $S^{1}$. The instability of the configuration 8 manifest itself in the lowest lying open string mode $T$, which becomes tachyonic. From the Chan-Paton factors of open strings that connect the two D branes, we know that the tachyon is charged with

\footnotetext{
${ }^{2}$ This result may sound quite counter-intuitive, because we are used to seeing one of the two $U(1)$ 's decouple from rest of dynamics. But we need to recall that the decoupling of the "center of mass" $U(1)$ follows from the combined effect of world-volume supersymmetry and translational invariance of string theory. On a $\mathrm{D} p$-anti- $\mathrm{D} p$ pair, supersymmetry is absent. See section 4 for detail.
} 
respect to a $U(1)$ gauge field, $A_{12}=A_{1}-A_{2}$, usually associated with the relative motion of the two branes. $A_{1}$ is the gauge field on D4 while $A_{2}$ is on anti-D4. Later it will turn out that the overall $U(1)$ gauge field $A_{S} \equiv A_{1}+A_{2}$ also plays a crucial role, but for the moment we will concentrate on $A_{12}$.

The tachyon rolls down to a true vacuum and condenses, $\langle T\rangle \neq 0$, but in the process its phase may acquire a winding number. Otherwise, the final state would correspond to the trivial vacuum of the string theory [2]. Because of its coupling to $A_{12}$, the winding number is accompanied by a localized magnetic flux of $A_{12}=A_{1}-A_{2}$ near $T=0$, and a magnetic vortex soliton is created. On the other hand, the world-volume Lagrangian contains a Chern-Simons term [9] of type,

$$
\int_{\mathrm{D} 4} C_{3} \wedge d A_{1}+\int_{\text {anti-D4 }} C_{3} \wedge d A_{2}=\int_{\mathrm{R}^{4+1}} C_{3} \wedge d\left(A_{1}-A_{2}\right),
$$

where $C_{3}$ is the Ramond-Ramond 3 -form tensor field. Note that, unlike the case of a D4 brane pair where the sum of gauge fields couples to $C_{3}$, the difference of the two gauge field couples to $C_{3}$. This is due to the opposite orientations carried by $\mathrm{D} 4$ and anti-D4.

Quantized magnetic flux generated by the winding number of $T$ is localized near $T=0$. Thus, integrating over the plane transverse to the flux line, the above coupling reduces to

$$
\int_{\{T=0\} \cap R^{4+1}=R^{2+1}} C_{3} \text {. }
$$

This is precisely how $C_{3}$ would have coupled to an ordinary D2 brane [10], from which one concludes that the resulting $(2+1)$-dimensional object must be a D2 brane [3]. Since D2 brane is the lightest object that carries the electric $C_{3}$ charge, and since the soliton does not seem to possess any other spacetime charge, it is a reasonable extrapolation.

Interestingly enough, at least part of spacetime supersymmetry is restored in the final state, be it the trivial vacuum or D2 branes. Although there is no sense in which the soliton was BPS with respect to the world-volume theory of D4-anti-D4, the soliton is actually BPS with respect to the spacetime supersymmetry. Despite the lack of supersymmetry of the effective field theory that produces the soliton, one can still reply on the soliton mass formula to be stable against continuous change of parameters. 
When we regard the D4-anti-D4 pair as a M5-anti-M5 pair wrapped on a compact circle $S^{1}$, the instability should persist. The only difference is that the tachyonic degrees of freedom must arise from open membranes that are stretched between the five-branes. The boundary of a membrane ending on five-branes is string-like, so the object that condenses is a tachyonic world-volume string. The open string tachyon on the D4-anti-D4 may be regarded as the tachyonic string wrapped on $S^{1}$. Given that D2 branes are nothing but transverse M2 branes, and given that the final state is preserved by restored spacetime supersymmetry, we conclude that a M5-anti-M5 pair annihilate and produce M2 branes as world-volume solitons. Let us see this phenomenon in M theory setting in more detail.

The M5 brane admits a single chiral 2-form $B^{+}$whose 3 -form field strength $\mathrm{H}^{+}$is restricted to be self-dual. The anti-M5 brane, on the other hand, is identical to the M5 brane, except for the reverse orientation. The orientation is in turn related to the chirality of the massless fermions and 2-form tensor. Anti-M5 thus admits anti-chiral $B^{-}$, whose field strength $H^{-}$is anti-self-dual. On an M5-anti-M5 system, the two fields combine to become a whole 2-form field, to be denoted by $B_{M N}$. When we wrap the M5-anti-M5 pair on a circle to make a D4-anti-D4 pair, this $B_{M N}$ reduces to a pair of vector fields. One is obtained from $B_{5 \mu}$, which we may identify with $\left(A_{12}\right)_{\mu}$. The remaining tensor $B_{\mu \nu}$ is dual to a vector in $(4+1)$ dimensions, which can be identified with the overall $U(1)$ vector $A_{S}$. (It may seem ambiguous which of the two $U(1)$ vectors $A_{12}$ and $A_{S}$ should be identified with, say, $B_{5 \mu}$. But actually, it is simply a matter of convention, since, by switching to the dual field strength $* H=H^{+}-H^{-}$in place of $H=H^{+}+H^{-}$, one effectively exchanges the longitudinal and the transverse parts.)

Because the 2-form $B$ is now unrestricted, its dynamics can be described by a Lagrangian. In particular, the Chern-Simon coupling (1) on the D4-anti-D4 pair must come from a similar coupling on the M5-anti-M5 pair wrapped on $S^{1}$, and the obvious conclusion we draw is that there is a term in the world-volume Lagrangian of the form [11],

$$
\int_{R^{5+1}=\mathrm{M} 5-\operatorname{anti-\mathrm {M}5}} C_{3} \wedge H
$$

As we will see in section 4, this coupling is also related to part of Born-Infeld actions of the D4 brane 
and of the anti-D4 brane, upon the compactification. Integrating over a localized and quantized magnetic flux $H$ on a transverse $R^{3}$, we find

$$
\int_{R^{2+1}} C_{3}
$$

Again this is how $C_{3}$ would have coupled to M2 brane, and so the localized and quantized magnetic $H$ flux behave as M2 brane.

So far, we are yet to say how such a soliton of $H$ flux is realized dynamically. Since the condensing charged objects are string-like, one would think that the low energy effective field theory is impossible to write down. But the essential ingredients of the soliton construction is not that mysterious. Recall that, when a gauge symmetry is broken spontaneously, there always exists an would-be Goldstone mode. In the above case of spontaneously broken $U(1)$ gauge theory, it is given by the phase $\theta$ of $T$, which is eaten up by the gauge field and give the latter an effective mass. The effective mass term appears as

$$
|T|^{2}\left(\partial_{\mu} \theta-A_{\mu}\right)^{2}
$$

and is gauge invariant on its own because a gauge transformation of $A$ is accompanied by a canceling shift of $\theta$. When the 1-form gauge field is replaced by 2 -form gauge field $B_{M N}$, one also should find a similarly gauge-invariant mass term that involves a new would-be Goldstone mode. A gauge transformation of 2-form $B$ is induced by 1-form, so the Goldstone boson itself should be 1-form. Call it $G_{M}$. Thus, the gauge-invariant mass term must look like [12],

$$
f^{2}\left(\partial_{M} G_{N}-\partial_{N} G_{M}-B_{M N}\right)^{2}
$$

where $f$ is some scalar field that is tachyonic and condenses to restore spacetime supersymmetry. However complicated is the underlying theory of M5 branes, this particular feature of condensation should be robust. Finally, the would-be Goldstone boson itself can carry a nontrivial topology, namely the topology of the complex line bundle associated with the connection $G$. Significance of this will become obvious in next section. 


\section{Condensation of Tachyonic String: a Toy Model}

Let us first recall how one obtains vortex solitons in $U(1)$ gauge theory. The condensation of charged particle can be treated using a simple effective field theory of a complex scalar field $T=|T| e^{i \theta}$. Let us point out the obvious and say that the phase $\theta$ is periodic in $2 \pi$. When it couples to a gauge field, the effective Lagrangian is

$$
\mathcal{L}=-\frac{1}{4} F_{\mu \nu}^{2}+\left(\partial_{\mu}|T|\right)^{2}+|T|^{2}\left(\partial_{\mu} \theta-e A_{\mu}\right)^{2}-U(|T|)
$$

In the decoupled theory, where $e \rightarrow 0$, one sees plane wave of charged field, $T$, which is promoted to charged particles upon quantization. In addition there exist some classical solitons. In the symmetric phase, one finds q-balls 13] for some $U(f)$, a nontopological soliton of net global $U(1)$ charge. In the broken phase, $\langle T\rangle \neq 0$, the soliton that one obtains is a global vortex, for which the periodic variable $\theta$ has a net winding number along some asymptotic circle. Since a continuous symmetry is broken, a Goldstone boson must be present and is identified with $\theta$.

When the gauge coupling $e$ is turned on, this global vortex becomes a soliton of finite energy that also carries a quantized magnetic flux: The gauge particle becomes massive by eating up the would-be Goldstone boson $\theta$. Because of this mass term, the asymptotic behavior of $A_{\mu}$ is now tied to that of $\partial_{\mu} \theta$, leading to a localized magnetic flux whenever $\theta$ has a winding number. Magnetic flux is quantized as

$$
\int_{R^{2}} F=\oint A=\frac{1}{e} \oint \partial \theta=\frac{2 \pi n}{e}
$$

The asymptotic form of $A$ is clearly a pure gauge, so the magnetic flux $F$ is localized near $|T|=0$. When we identify $A$ as the relative $U(1)$ gauge field $A_{12}$, this vortex soliton in the broken phase is exactly what Sen identified as the D0 brane obtained from a D2-anti-D2 pair.

One can ask if there is an analogous effective description when charged string condenses? First of all, 1-form gauge field $A_{\mu}$ is promoted to 2-form gauge field $B_{M N}$, so naturally the would-be Goldstone boson $\theta$ is also promoted to a 1 -form, $G_{M}$. Denote their field strengths by $H_{M N P}$ and $K_{M N}$, respectively. One can imagine that the relevant string field is defined over a loop space and 
that the 1-form $G$ comes from a factor of it,

$$
e^{i \oint G}
$$

The phase factor $\exp (i \oint G)$ must be single-valued in the loop space, and this quantizes possible net $K$ flux along any closed surface $\Sigma$;

$$
\oint_{\Sigma} K=2 \pi n
$$

where $n$ is an integer. One possible generalization of the effective Lagrangian above is then,

$$
\mathcal{L}=\frac{1}{12}\left(H_{M N P}\right)^{2}+\frac{1}{2}\left(\partial_{M} f\right)^{2}-m(f)^{2}\left(K_{M N}-e B_{M N}\right)^{2}-U(f)
$$

where $f$ is a macroscopic version of some modulus field associated with the factor $e^{i \oint G}$. $U$ and $m$ are functions of $f$ that must be determined microscopically. We wish to argue that this Lagrangian contains many qualitative features one would expect from string condensation.

Suppose $e=0$ for the moment. The physics of the symmetric phase is that of a strongly interacting QED with "gauge field" $G_{M}$, coupled to a real scalar $f$. Again, the symmetric phase $(\langle f\rangle \neq 0)$ may contain nontopological soliton which now consists of a flux tube of $K_{M N}$, given an appropriate form of $U(f)$. The existence argument is almost identical to the q-ball case [14]. 3] In other words, the theory contains a solitonic string in the symmetric phase, which couples to $B$ as a fundamental string would when we turn $e$ back on. This is one justification for using the above as a toy model for the string condensation.

Let us keep $e \neq 0$. When the potential $U$ becomes tachyonic, the theory settles down in the broken phase, $\langle f\rangle \neq 0$. Since the would-be Goldstone boson is now a vector, a possible topological defect

\footnotetext{
${ }^{3}$ Suppose that the electric field is spread evenly on transverse volume $V$ and that the scalar field is constant $f=v$ in this region. The total flux is approximately $\Phi=f^{2} E_{01} V$. The total energy per unit length of the string will be then

$$
\mathcal{E}=\frac{\Phi^{2}}{2 v^{2} V}+V U(f)
$$

When $V^{2}=\Phi^{2} /\left(2 f^{2} U(f)\right)$, the minimum of this energy density would be obtained with value

$$
\mathcal{E}=\Phi \sqrt{\frac{2 U(f)}{f^{2}}} .
$$

Once there is the minimum of $2 U(f) / f^{2}$ away from the symmetric phase, there exists a stable q-bundle.
} 
is that of a Dirac monopole. The defect imposes a boundary condition at infinity of type,

$$
\oint_{S^{2}} K=2 \pi n
$$

For $n \neq 0$, the 1 -form field $G_{M}$ requires more than one coordinate patches, and, if we associate $G$ with a line bundle on $S^{2}$, the integer $n$ would be the first Chern class of that line bundle.

In this asymmetric phase, the gauge-invariant mass term for $B$ tells us that the asymptotic behavior of $B$ is tied to that of $K$. The flux of $H$ is then quantized as

$$
\int_{R^{3}} H=\oint_{S^{2}} B=\frac{1}{e} \oint_{S^{2}} K=\frac{2 \pi n}{e}
$$

The asymptotic form of $B \simeq K$ is pure gauge, since $d K=0$, so the $H=d B$ flux is again localized near $f=0$, just as in the vortex soliton, except that now the resulting solitonic object is of co-dimension three. Given the topological coupling (3), this soliton is interpreted as M2 brane.

One may worry whether a finite mass soliton is possible in such a theory, let alone whether the soliton has the right mass to be a M2 brane. While one does not know the detailed form of the soliton, it is relatively easy to see how possible divergences in energy density are avoided. At large distances, it is the behavior of $H^{2}$ that could be troublesome. However, the asymptotics, $B \sim K$, tells us that $H$ decays as $1 / r^{3}$, so the energy density contribution $H^{2}$ scales as $1 / r^{6}$. In $(5+1)$ dimensions, the integrated energy is convergent. Near the soliton cores, where the match between $B$ and $K$ need not hold any more, it is vanishing $f$ and thus $m(f)$ that should come to the rescue. By having $m(f) \rightarrow 0$ sufficiently fast, we do find a finite core energy. For instance, $m(f) \sim f^{2}$ near $f=0$ will do. (Although, since this effective Lagrangian governs long distance dynamics at best, it is not meaningful to ask exactly what $m(f)$ is for small $f$. A better prescription might be to introduce a short distance cut-off.)

\section{Fundamental Strings from $\mathrm{D} p$-anti-D $p$; Confinement of $A_{S}$}

Discussions in previous sections apply to other cases. As we observed above, NS5 brane of type IIA theory has essentially the same low energy behavior as M5 brane, so the same analysis will show 
that D2 branes are produced when an NS5 brane annihilates an anti-NS5 brane. Another way to see this is to compactify M theory such that neither the M5's nor the resulting M2 is wrapped on $S^{1}$.

One can consider an alternate compactification of M5-anti-M5 to a string theory configuration, in such a way that the resulting M2 brane is wrapped on $S^{1}$ and becomes a fundamental string. This M2 would be lying on M5-anti-M5 world-volume, so the M5's also have to be wrapped on $S^{1}$ and become D4's. It follows that fundamental strings are produced when a D4 brane annihilates an anti-D4 brane. One naturally wonders how such co-dimension-three objects are possible when D4 brane theory is an ordinary gauge theory.

Expanding Born-Infeld action of the D4 brane and the anti-D4 brane, one finds terms,

$$
\int_{\mathrm{D} 4}\left\langle\mathcal{B}, d A_{1}\right\rangle+\int_{\text {anti-D } 4}\left\langle\mathcal{B}, d A_{2}\right\rangle=\int_{R^{4+1}}\left\langle\mathcal{B}, d\left(A_{1}+A_{2}\right)\right\rangle
$$

where $\mathcal{B}$ is NS-NS 2-form field pulled back to the common world-volume. The two gauge fields are summed in this case, because the inner product $\langle$,$\rangle is insensitive to the orientation of the world-$ volume. Clearly it is the electric flux of the overall $U(1)$ gauge field $A_{S} \equiv A_{1}+A_{2}$ that would generate the fundamental string charge of $\mathcal{B}$.

On the other hand, from the above discussions of M5-anti-M5, we know that open D2 ending on a D4-anti-D4 pair provides string-like tachyonic objects. This object is magnetically charged with respect to the world-volume gauge fields $A_{1}$ and $A_{2}$. More specifically, it couples minimally to $B^{\prime} \equiv B_{1}^{\prime}-B_{2}^{\prime}$ when we dualize the gauge vectors $A_{i}$ 's to gauge 2 -form $B_{i}^{\prime \prime}$ s. This actually implies that it carries a magnetic charge of $A_{S}$, rather than that of $A_{12}$. As we saw in section 3 , the Hodge dual operator on anti-D4 has an extra sign with respect to the one on D4, owing to the reverse orientation. This introduces a sign when we dualize the gauge field, so we find that

$$
d\left(B_{1}^{\prime}-B_{2}^{\prime}\right)=* d\left(A_{1}+A_{2}\right)
$$

where the Hodge dual operator $*$ is defined with respect to D4. Thus, the tachyonic string is indeed magnetically charged with respect to $A_{S}=A_{1}+A_{2}$. 
Once this tachyonic string condenses, the $U(1)$ gauge field $A_{S}$ is confined by the dual Higgs mechanism. The only remnant of $A_{S}$ is the confined electric flux string. The world-volume coupling (16) induces $\mathcal{B}$ charge on $A_{S}$ flux, so the confined flux string is the fundamental string of type IIA theory.

Let us see how the gauge-invariant mass term for $B^{\prime}$ arises from $\mathrm{M}$ theory picture. It must be present if dual Higgs mechanism is to work for the overall $U(1)$. We already noted that, upon the compactification on a circle, the M5-anti-M5 world-volume field $B$ reduces to a pair of vector fields on D4-anti-D4. Having identified the longitudinal part $B_{5 \mu}$ as the relative $U(1)$ vector $\left(A_{12}\right)_{\mu}$, we know that the overall $U(1) A_{S}$ must be dual to the transverse part $B_{\mu \nu}$. That is, $B^{\prime}$ is nothing but the part of $B$ parallel to the D4-anti-D4 world-volume. The would-be Goldstone boson $G_{M}$ also reduces to a single angle $\theta=G_{5}$ and a vector $a_{\mu}=G_{\mu}$. The $B$ mass term (6) on M5-anti-M5 is then split into two pieces. The first,

$$
\left(\left(A_{12}\right)_{\mu}-\partial_{\mu} \theta\right)^{2}
$$

is the mass term for $\left(A_{12}\right)$, while the second

$$
\left(B_{\mu \nu}^{\prime}-\partial_{\mu} a_{\nu}+\partial_{\nu} a_{\mu}\right)^{2}
$$

is the mass term for the dual photon $B^{\prime}$.

This identification also provides an alternate check on the all-important topological coupling (3) on M5-anti-M5. A dimensional reduction of the coupling (3), when 3-form $C_{3}$ is aligned along $S^{1}$ and becomes NS-NS 2-form $\mathcal{B}$, gives us

$$
\int_{R^{4+1}} \mathcal{B} \wedge d B^{\prime}=\int_{R^{4+1}} \mathcal{B} \wedge d\left(B_{1}^{\prime}-B_{2}^{\prime}\right)=\int_{R^{4+1}}\left\langle\mathcal{B}, d\left(A_{1}+A_{2}\right)\right\rangle
$$

which is, as we saw in Eq. (16), part of well-established Born-Infeld actions of the D4 brane and of the anti-D4 brane.

Although there is no analogous $\mathrm{M}$ theory picture for other $\mathrm{D} p$-anti- $\mathrm{D} p$ pairs, it is rather clear that a generalization of this mechanism to arbitrary $\mathrm{D} p$-anti- $\mathrm{D} p$ must also exist. Open $\mathrm{D}(p-2)$ branes ending on the pair must provide a $(p-2)$-dimensional tachyonic objects, which couples to 
the overall $U(1)$ gauge field $A_{S}$ magnetically. The subsequent condensation confines the unbroken $U(1)$, and the fundamental string is realized as its electric flux string. One example where this is most clearly seen is the case of D3-anti-D3, where $S L(2, Z)$ U-duality maps fundamental strings to D-strings and vice sersa, while keeping D3's invariant.

\section{Summary}

We have observed how the Higgs mechanism of world-volume 2-form field $B$ produces co-dimension three solitonic byproducts on M5-anti-M5, type IIA NS5-anti-NS5, and D4-anti-D4 systems. They are interpreted as M2 branes, D2 branes, and fundamental strings, respectively. In particular, the fundamental string that emerges from a D4-anti-D4 pair, is nothing but the confined electric flux string of the overall $U(1)$ which remains unbroken upon condensation of the open string tachyon.

Generally, there exist a pair of tachyonic objects when a $\mathrm{D} p$ brane is coincident with an anti-D $p$ brane. One is perturbative in origin and couples to the relative $U(1)$ electrically, while the other is nonperturbative and couples to the overall $U(1)$ magnetically. The combined electric and magnetic Higgs phenomena introduce mass gaps to both $U(1)$ gauge sectors. The only remnants are $\mathrm{D}(p-2)$ branes and fundamental strings, which are realized as "solitons" on the common $(p+1)$-dimensional world-volume. In particular, this neatly addresses the question raised by Witten [4] of how the unbroken $U(1)$ becomes invisible on the annihilated $\mathrm{D} p$-anti- $\mathrm{D} p$ world-volume.

\section{Acknowledgment}

The author benefited from conversations with several visitors to Korea Institute for Advanced Study (KIAS). Kentaro Hori's probing questions lead to many of the insights of this paper, while Mans Henningson's expertise on M5 brane was very helpful. Most of all, the author is grateful to Kimyeong Lee who explained how a Higgs mechanism of 2-form tensor should be treated, and who is largely responsible for content of section 3. The author also thanks Miao Li for critical comments. This work is supported in part by National Science Foundation of the United States. 


\section{References}

[1] A. Sen, J. High Energy Phys. 9808 (1998) 010, hep-th/9805019.

[2] A. Sen, J. High Energy Phys. 9808 (1998) 012, hep-th/9805170.

[3] A. Sen, J. High Energy Phys. 9809 (1998) 023, hep-th/9808141.

[4] E. Witten, hep-th/9810188.

[5] P. Horava, hepth/9812135.

[6] H. Garcia-Compean, hep-th/9812226.

[7] S. Gukov, hep-th/9901042.

[8] M. Green, Phys. Lett. B329 (1994) 435, hep-th/9403040; T. Banks and L. Susskind, hepth/9511194; M. Green and M. Gutperle, Nucl.Phys. B476 (1996) 484, hep-th/9604091; G. Lifschytz, Phys. Lett. B388 (1996) 720, hep-th/9604156; V. Periwal, hep-th/9612215.

[9] M. Li, Nucl.Phys. B460 (1996) 351, hep-th/9510161; M. Green, J.A. harvey, G. Moore, Class.Quant.Grav. 14 (1997) 47, hep-th/9605033.

[10] J. Polchinski, Phys. Rev. Lett. 75 (1995) 4724, hep-th/9510017.

[11] E. Witten, J.Geom.Phys. 22 (1997) 103-133, hep-th/9610234.

[12] S. Rey, Phys. Rev. D40 (1989) 3396.

[13] S. Coleman, Nucl. Phys. B262 (1985) 263; Erratum-ibid.B269 (1986) 744.

[14] K. Lee, private communication. 